\title{
A Time Domain Point Source Method for Inverse Scattering by Rough Surfaces
}

\author{
C.D. Lines \\ Department of Mathematical Sciences, Brunel University, \\ Uxbridge, Middlesex, UB8 3PH, U.K. \\ S.N. Chandler-Wilde Department of Mathematics, University of Reading, \\ Whiteknights, PO Box 220, Berkshire RG6 6AX, U.K.
}

\begin{abstract}
In this paper we propose a new method to determine the location and shape of an unbounded rough surface from measurements of scattered electromagnetic waves. The proposed method is based on the point source method of Potthast (IMA J.Appl.Math., 61:119-140, 1998) for inverse scattering by bounded obstacles. We propose a version for inverse rough surface scattering which can reconstruct the total field when the incident field is not necessarily time harmonic. We present numerical results for the case of a perfectly conducting surface in TE polarization, in which case a homogeneous Dirichlet condition applies on the boundary. The results show great accuracy of reconstruction of the total field and of the prediction of the surface location.
\end{abstract}

\section{Introduction}

The determination of the elevation of the ground, sea surface, or sea bed are basic problems in remote sensing by sonar or radar. To model the scattering problem, boundary or domain integral representations of the scattered field are commonly used and the inverse problem linearised by a Born or Kirchhoff approximation to the field on the scatterer, or the inversion carried out by interpreting time of travel data (e.g. [8, 9, 29, 12, 14, 27, 28, 30]).

Recently a range of mathematical methods for solving the nonlinear inverse problem, determining the support of a bounded scattering obstacle directly from measurements of the scattered field, have been proposed, including factorization and linear sampling methods $[11,16,10,2,1]$ and the method of singular sources of Potthast [24]. These methods have in common: (i) the solution of first kind linear integral equations; and (ii) that they require measurements of a large quantity of data. Illustrating the second point, consider the application of the linear sampling method to the detection of a buried obstacle below a flat interface [10]. The data required are measurements of the field at every point on a two-dimensional finite horizontal grid above the interface for a point source at every point on the same grid. For unbounded periodic surfaces a version of the factorization method has recently been proposed by Arens \& Kirsch [2], and see [13] for another method for the same inverse scattering problem.

In this paper we explore the application, to detect the position of an unbounded surface, of the point source method proposed by Potthast [23, 24] for inverse scattering by bounded obstacles. This method has more modest data requirements (as do other somewhat related methods recently proposed by Potthast et al [26, 25] and by Ikehata [15] - see [25] for a more detailed review and classification). In our two-dimensional version of the point source method, a single time harmonic point source transmitter of an electromagnetic field is located above the surface to be located and the total field produced is measured on a finite horizontal line, 
also above the ground surface. Our version of the point source method is a procedure for approximating the total field at all points above the ground surface from these few measurements, via solution of an (ill-posed) linear Fredholm integral equation of the first kind. We briefly explain the justification for this method (for more detail see $[18,19]$ ). We then propose a time domain point source method to reconstruct the total electric field when the incident wave is not necessarily time harmonic. In particular, in the numerical experiments we carry out, we have chosen an incident pulse that is similar to those arising in ground penetrating radar applications. We show numerical examples for the simplest case, when the boundary is perfectly conducting and the incident field is in TE polarization, so that a homogenous Dirichlet condition applies on the boundary. We reconstruct the total field above the surface and also locate the position of the boundary as the curve along which the reconstructed total field is minimal.

\section{The Surface Scattering Problem}

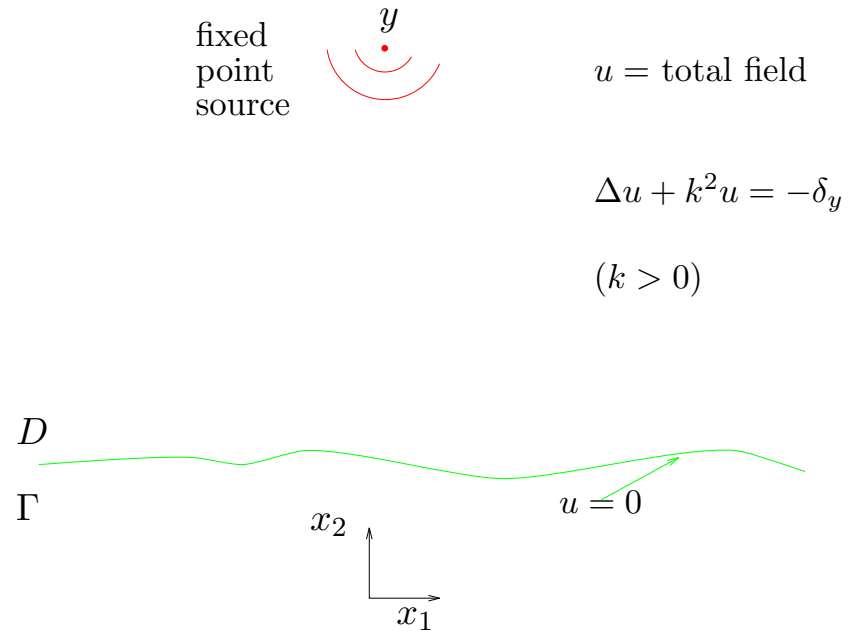

Figure 1: The direct problem for the total field $u=G(\cdot, y)$.

To simplify the problem, and since the rigorous analysis of $3 D$ problems of scattering by unbounded rough surfaces is very much in its infancy, we consider the $2 \mathrm{D}$ case in this paper. Adopting a Cartesian coordinate system $\mathrm{Ox}_{1} x_{2} x_{3}$, the incident field and scattering surface are invariant in the $x_{3}$ direction. We will present results later for the case of an impulsive source, but for the moment consider the case when the incident electromagnetic field is that from a time harmonic ( $e^{-i \omega t}$ time dependence) monopole source in TE polarization, and the scattering surface is perfectly conducting. Let $C^{1,1}(\mathbb{R})$ denote the set of functions $f: \mathbb{R} \rightarrow \mathbb{R}$ which are bounded and continuously differentiable, with Lipschitz continuous derivative, so that, for some constant $C>0$,

$$
\left|f^{\prime}(s)-f^{\prime}(t)\right| \leq C|s-t|, \quad s, t \in \mathbb{R} .
$$

We suppose that the scattering surface is given by

$$
\Gamma=\left\{\left(x_{1}, f\left(x_{1}\right)\right) \mid x_{1} \in \mathbb{R}\right\},
$$


for some $f \in C^{1,1}(\mathbb{R})$ and hence, for some constants $f_{-}, f_{+} \in \mathbb{R}$,

$$
f_{-} \leq f\left(x_{1}\right) \leq f_{+}, \quad x_{1} \in \mathbb{R} .
$$

The electromagnetic field satisfies the Maxwell equations in the region $D=\left\{\left(x_{1}, x_{2}\right) \mid x_{2}>\right.$ $\left.f\left(x_{1}\right)\right\}$ above $\Gamma$. With these assumptions, and using the notations $x=\left(x_{1}, x_{2}\right), y=\left(y_{1}, y_{2}\right)$ throughout, the electric field at $\left(x_{1}, x_{2}, x_{3}\right)$ when the source is at $y \in D$ is $E=(0,0, G(x, y))$, where $u:=G(\cdot, y)$ satisfies the inhomogenous Helmholtz equation,

$$
\Delta u+k^{2} u=-\delta_{y}
$$

in $D$, where $k=\omega / c$ and $c$ is the wave speed (see Figure 1). Further, $u=0$ on $\Gamma$ and $u$ satisfies the Sommerfeld radiation conditions (equations (7) below).

Let

$$
\Phi(x, y):=\frac{i}{4} H_{0}^{(1)}(k|x-y|), \quad x, y \in \mathbb{R}^{2}, x \neq y .
$$

Then $\Phi(\cdot, y)$ is the unique solution of (3) in $\mathbb{R}^{2}$ which satisfies the Sommerfeld radiation conditions, i.e. $\Phi(\cdot, y)$ is the incident field, the electric field in the absence of the scattering surface. Let $U^{s}(x, y):=G(x, y)-\Phi(x, y)$ denote the scattered part of the electric field. Then $v:=U^{s}(\cdot, y)$ satisfies the following Dirichlet boundary value problem (the direct problem): find $v \in C^{2}(D) \cap C(\bar{D})$ such that

$$
\begin{array}{r}
\Delta v+k^{2} v=0 \quad \text { in } D, \\
v=-\Phi(., y) \quad \text { on } \Gamma, \\
v(x)=O\left(r^{-\frac{1}{2}}\right), \\
\frac{\partial v(x)}{\partial r}-i k v(x)=o\left(r^{-\frac{1}{2}}\right),
\end{array}
$$

as $r:=|x| \rightarrow \infty$, uniformly in $\hat{x}=x /|x|$. From [7] and [6, Theorem 5.1], we have that this boundary value problem has exactly one solution.

For $h \in \mathbb{R}$ let $U_{h}:=\left\{\left(x_{1}, x_{2}\right) \mid x_{2}>h, x_{1} \in \mathbb{R}\right\}$ and define

$$
G_{1, h}(x, y):=\Phi(x, y)+\Phi\left(x, y_{h}^{\prime}\right)+P\left(k\left(x-y_{h}^{\prime}\right)\right), \quad x, y \in \overline{U_{h}}, \quad x \neq y,
$$

where

$$
P(z):=\frac{e^{i|z|}}{\pi} \int_{0}^{\infty} \frac{t^{-\frac{1}{2}} e^{-|z| t}(1+\gamma(1+i t))}{\sqrt{t-z i}(t-i(1+\gamma))^{2}} \mathrm{dt}, \quad z \in \overline{U_{0}},
$$

with $\gamma:=z_{2} /|z|$, and $y_{h}^{\prime}=\left(y_{1}, 2 h-y_{2}\right)$ the reflection of $y$ in $\Gamma_{h}:=\partial U_{h}$. It is shown in $[4,3]$ that $P \in C\left(\overline{U_{0}}\right) \cap C^{\infty}\left(\overline{U_{0}} \backslash\{0\}\right)$ and satisfies the Helmholtz equation and Sommerfeld radiation conditions (with $k=1$ ) in $U_{0}$. Further, if $v:=G_{1, h}(., y)$ we have that (see $[4,3]$ )

$$
\begin{aligned}
\Delta v+k^{2} v & =-\delta_{y} & & \text { in } U_{h} \\
\frac{\partial v}{\partial x_{2}}+i k v & =0 & & \text { on } \Gamma_{h},
\end{aligned}
$$

$$
\left.\begin{array}{ll}
v(x) & =O\left(r^{-\frac{1}{2}}\right) \\
\frac{\partial v(x)}{\partial r}-i k v(x) & =o\left(r^{-\frac{1}{2}}\right)
\end{array}\right\} \text { as } r:=|x| \rightarrow \infty, \text { uniformly in } \hat{x}
$$


Thus $G_{1, h}$ is the Green's function for the Helmholtz equation in the half-plane $U_{h}$ which satisfies the impedance boundary condition (6). From [5], $G_{1, h}$ satisfies the bound

$$
\left|G_{1, h}(x, y)\right| \leq C\left(1+x_{2}-h\right)\left(1+y_{2}-h\right)|x-y|^{-\frac{3}{2}}, \quad x, y \in \overline{U_{h}}, \quad x \neq y,
$$

for some constant $C>0$ depending only on $k$, so that $G_{1, h}$ decays faster than required by the Sommerfeld radiation conditions if $x_{2}$ and $y_{2}$ stay close to the boundary $\Gamma_{h}$.

Echoing earlier notation we define

$$
U_{1}^{s}(x, y):=G(x, y)-G_{1, h}(x, y), \quad x, y \in \bar{D},
$$

so that $U_{1}^{s}(\cdot, y)$ is the solution to the Dirichlet BVP for boundary data $-G_{1, h}(\cdot, y)$ on $\Gamma$.

\section{The Point Source Method}

Let $A>0, H>\sup f$, and let $\gamma^{*}$ denote the finite horizontal line

$$
\gamma^{*}=\left\{\left(x_{1}, H\right)|| x_{1} \mid \leq A\right\} .
$$

Then the first inverse problem we will consider is as follows.

The Inverse Problem. Given measurements of the total field $G(x, z)$, for $x \in \gamma^{*}$ and a single source position $z \in D \backslash \gamma^{*}$, determine $f$, i.e. the location of the infinite surface $\Gamma$.

Remark 3.1 It is an open question whether this inverse problem is uniquely solvable. If the incident field is replaced by a plane wave then the inverse problem can have more than one solution as shown by the following simple example. Take the incident field to be the plane wave $\exp \left(-i k x_{2}\right)$, and choose $p<q<H$ with $q-p$ a multiple of $\pi / k=\lambda / 2$, where $\lambda=2 \pi / k$ is the wavelength. Then the flat surfaces $\Gamma=\Gamma_{p}$ and $\Gamma=\Gamma_{q}$ produce the same total field, namely $\exp \left(-i k x_{2}\right)-\exp \left(i k\left(x_{2}-2 p\right)\right)$, and so the same measurements on $\gamma^{*}$.

The method we will formulate for computing a solution to this inverse problem is based on the point source method of Potthast $[23,24]$ for scattering by bounded obstacles. Our point source method is a method to construct, from the measured data, namely $G(x, z)$ for $x$ on $\gamma^{*}$, an approximation $G^{\alpha}(x, z)$ to the total field $G(x, z)$ for all $x \in D$. Since $G(\cdot, z)$ vanishes on $\Gamma$, and provided $G(\cdot, z)$ does not vanish on other curves in $D$, a possibility is to look for the surface as a minimum of $\left|G^{\alpha}(\cdot, z)\right|$, and this is the approach adopted.

The first step in constructing $G^{\alpha}(\cdot, z)$ is to note the reciprocity result shown in [18, Theorem 3.1.4], that

$$
G\left(x^{*}, z\right)=G\left(z, x^{*}\right), \quad x^{*}, z \in D \text {. }
$$

It follows that we can proceed by constructing an approximation to $G\left(z, x^{*}\right)$ for $x^{*} \in D$. Suppose $x^{*} \in D \backslash\{z\}$ and $f_{0} \in C^{1,1}(\mathbb{R})$ with $f_{0}(0)<0$, and define $f_{x^{*}} \in C^{1,1}(\mathbb{R})$ by $f_{x^{*}}\left(x_{1}\right):=$ $f_{0}\left(x_{1}-x_{1}^{*}\right)+x_{2}^{*}, x_{1} \in \mathbb{R}$, and let

$$
\Gamma^{x^{*}}:=\left\{\left(x_{1}, f_{x^{*}}\left(x_{1}\right)\right) \mid x_{1} \in \mathbb{R}\right\} .
$$

We assume that values $f_{-}$and $f_{+}$for which (2) holds are known and define $\epsilon_{+}:=\sup _{x_{1} \in \mathbb{R}} f_{0}\left(x_{1}\right)$ and $\epsilon_{-}:=-\inf _{x_{1} \in \mathbb{R}} f_{0}\left(x_{1}\right)$. Then $\Gamma$ lies between the lines $\Gamma_{f_{-}}$and $\Gamma_{f_{+}}$and it is enough, for 


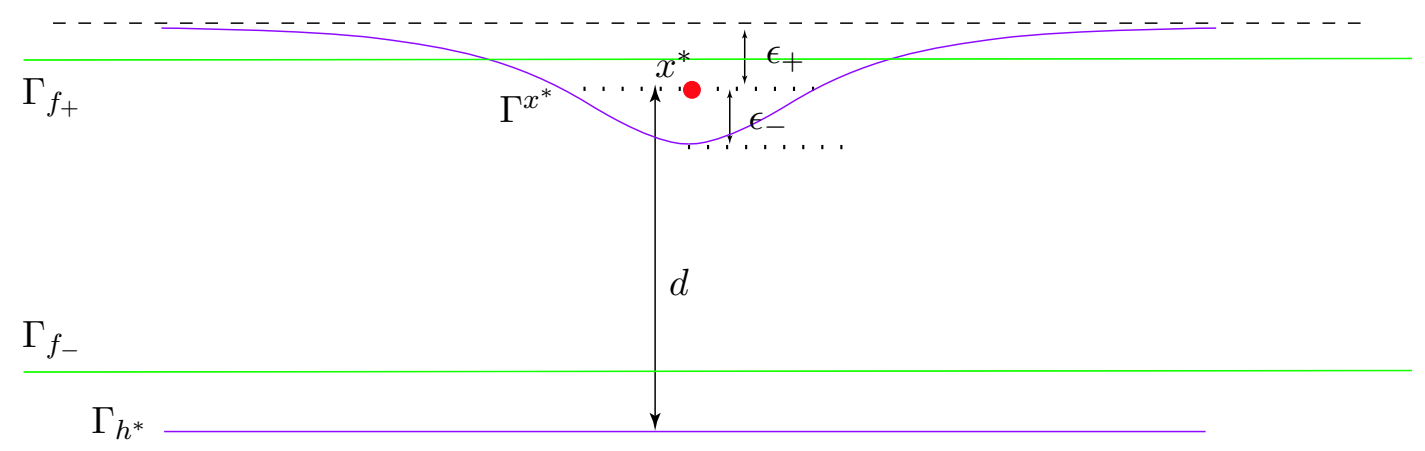

Figure 2: Geometry for equation (12).

the purpose of locating $\Gamma$, to reconstruct $G\left(x^{*}, z\right)=G\left(z, x^{*}\right)$ for $x^{*} \in \bar{U}_{f_{-}} \backslash U_{f_{+}}$, i.e. for $f_{-} \leq x_{2}^{*} \leq f_{+}$. We assume further that $f_{0}$ and $H$ are chosen so that $H>f_{+}+\epsilon_{+}$. Then, for $f_{-} \leq x_{2}^{*} \leq f_{+}, \Gamma^{x^{*}}$ lies below $\Gamma_{H} \supset \gamma^{*}$. Choose $d>\max \left(f_{+}-f_{-}, \epsilon_{-}\right)$and let $h^{*}:=x_{2}^{*}-d$ so that $h^{*}<f_{-}$and $\Gamma^{x^{*}} \subset U_{h^{*}}$, for $f_{-} \leq x_{2}^{*} \leq f_{+}$(see Figure 2).

Consider the first kind integral equation

$$
\int_{\gamma^{*}} G_{1, h^{*}}(x, y) \phi_{x^{*}}(y) \operatorname{ds}(y)=g_{x^{*}}(x):=G_{1, h^{*}}\left(x, x^{*}\right), \quad x \in \Gamma^{x^{*}},
$$

where $G_{1, h^{*}}$ is defined by equation (5) and $f_{-} \leq x_{2}^{*} \leq f_{+}$. This equation can be written in operator form as

$$
K \phi_{x^{*}}=g_{x^{*}}
$$

where the integral operator $K$ is defined by

$$
K \psi(x)=\int_{\gamma^{*}} G_{1, h^{*}}(x, y) \psi(y) \mathrm{ds}(y), \quad x \in \Gamma^{x^{*}},
$$

for $\psi \in L^{2}\left(\gamma^{*}\right)$. Since the Green's function $G_{1, h^{*}}$ satisfies the bound (8) it follows that $K: L^{2}\left(\gamma^{*}\right) \rightarrow L^{2}\left(\Gamma^{x^{*}}\right)$ and is bounded. It is shown in $[18,19]$ that $g_{x^{*}}$ is not in the range of $K$ so that (12) does not have a solution. However, it is also shown in [18, 19] that $K$ has dense range (and is injective) so that we can find a function $\phi_{x^{*}}^{\alpha} \in L^{2}\left(\gamma^{*}\right)$ which solves (12) approximately to arbitrary accuracy.

A standard method to compute such a solution is Tikhonov regularisation, in which $\phi_{x^{*}}^{\alpha}$ is found as the unique solution of

$$
\alpha \phi_{x^{*}}^{\alpha}+K^{*} K \phi_{x^{*}}^{\alpha}=K^{*} g_{x^{*}}
$$

where $K^{*}$ is the adjoint of $K$ and $\alpha>0$ is the regularisation parameter. As $g_{x^{*}}$ is not in the range of $K$, necessarily $\left\|\phi_{x^{*}}^{\alpha}\right\|_{L^{2}\left(\gamma^{*}\right)} \rightarrow \infty$ as $\alpha \rightarrow 0$. On the other hand, since the range of $K$ 
is dense, the standard theory of Tikhonov regularisation (see [18, Theorem 2.2.4]) guarantees that the residual $\left\|K \phi_{x^{*}}^{\alpha}-g_{x^{*}}\right\|_{L^{2}\left(\Gamma^{x^{*}}\right)} \rightarrow 0$ as $\alpha \rightarrow 0$.

Thus, defining the residual in (12) by

$$
u(x):=\int_{\gamma^{*}} G_{1, h^{*}}(x, y) \phi_{x^{*}}^{\alpha}(y) \operatorname{ds}(y)-G_{1, h^{*}}\left(x, x^{*}\right), \quad x \in \bar{U}_{h^{*}} \backslash\left\{x^{*}\right\},
$$

we can make $u$ as small as we like in the $L^{2}$ norm on $\Gamma^{x^{*}}$ by choosing $\alpha$ small enough. Since we take $f_{-} \leq x_{2}^{*} \leq f_{+}$, the line $\Gamma_{h^{*}}$ lies below $\Gamma^{x^{*}}$. Let $G^{x^{*}}$ denote the unbounded waveguide/duct-like region between $\Gamma^{x^{*}}$ and $\Gamma_{h^{*}}$. As discussed in detail in [18, 19], $u$ satisfies the Helmholtz equation in $G^{x^{*}}$ and the homogeneous impedance boundary condition (6) on $\Gamma_{h^{*}}$. It is shown in $[18,19]$ that this implies that if $u$ is small in the $L^{2}$ norm on $\Gamma^{x^{*}}$ then it is small in the supremum norm in $G_{\epsilon}^{x^{*}}$, for every $\epsilon>0$, where $G_{\epsilon}^{x^{*}}$ is $G^{x^{*}}$ with an $\epsilon$ neighbourhood of $\Gamma^{x^{*}}$ removed. Thus, if $x^{*}$ is a point in $D$ for which $\Gamma$ lies below $\Gamma^{x^{*}}$, so that $\Gamma^{x^{*}} \subset G^{x^{*}}$, then $u$ is small in the supremum norm on $\Gamma$ if $u$ is small in the $L^{2}$ norm on $\Gamma^{x^{*}}$.

Replacing $G_{1, h^{*}}(x, \cdot)$ with $U_{1}^{s}(x, \cdot)$ in (15) we define

$$
w(x):=\int_{\gamma^{*}} U_{1}^{s}(x, y) \phi_{x^{*}}^{\alpha}(y) \operatorname{ds}(y)-U_{1}^{s}\left(x, x^{*}\right), \quad x \in \bar{D} .
$$

Then $w$ is the solution to the Dirichlet BVP in $D$ with boundary data $w=-u$ on $\Gamma$ and so, by continuous dependance results for this boundary value problem in [7], if $u$ is small in the supremum norm on $\Gamma$ then $w$ is small (in a weighted supremum norm) in $D$, in particular $w$ is small at $z$, in other words

$$
U_{1}^{s}\left(z, x^{*}\right) \approx \int_{\gamma^{*}} U_{1}^{s}(z, y) \phi_{x^{*}}^{\alpha}(y) \mathrm{ds}(y) .
$$

Now, using the reciprocity relation, the definition of $U_{1}^{s}(z, y)$ and (16), it follows from the above discussion that, if $\alpha$ is chosen small enough, then

$$
\begin{aligned}
G\left(x^{*}, z\right) & =G\left(z, x^{*}\right) \\
& =G_{1, h^{*}}\left(z, x^{*}\right)+U_{1}^{s}\left(z, x^{*}\right) \\
& \approx G_{1, h^{*}}\left(z, x^{*}\right)+\int_{\gamma^{*}} U_{1}^{s}(z, y) \phi_{x^{*}}^{\alpha}(y) \mathrm{ds}(y) \\
& =G_{1, h^{*}}\left(z, x^{*}\right)+\int_{\gamma^{*}}\left(G(y, z)-G_{1, h^{*}}(z, y)\right) \phi_{x^{*}}^{\alpha}(y) \mathrm{ds}(y) .
\end{aligned}
$$

In this last expression, the values $G(y, z), y \in \gamma^{*}$, are the measured data. Based on this expresssion we define, as our approximation to $G\left(x^{*}, z\right)$, the quantity

$$
G^{\alpha}\left(x^{*}, z\right):=G_{1, h^{*}}\left(z, x^{*}\right)+\int_{\gamma^{*}}\left(G(y, z)-G_{1, h^{*}}(z, y)\right) \phi_{x^{*}}^{\alpha}(y) \operatorname{ds}(y), x^{*} \in \bar{U}_{f_{-}} \backslash U_{f_{+}} .
$$

Thus our point source method is: given a source position $z$, and the total field $G(y, z)$, for $y \in \gamma^{*}$, compute $G^{\alpha}\left(x^{*}, z\right)$ for $x^{*} \in \bar{U}_{f_{-}} \backslash U_{f_{+}}$, and look for the location of the surface as the minimum of $\left|G^{\alpha}\left(x^{*}, z\right)\right|$.

By arguments which have been sketched briefly above the following bound on the error in the point source method approximation is shown in $[18,19]$. In this theorem, for $f \in C^{1,1}(\mathbf{R})$, $\|f\|_{C^{1,1}(\mathbf{R})}$ denotes the norm $\|f\|_{C^{1,1}(\mathbf{R})}:=\|f\|_{\infty}+\left\|f^{\prime}\right\|_{\infty}+C_{\min }$, where $C_{\min }$ is the smallest value of $C$ for which (1) holds. 
Theorem 3.1 For every $\epsilon>0$ and $c>0$ there exists $C>0$, dependent only on $k, f_{0}, d, \epsilon, z_{2}-$ $\inf f$, and $c$, such that, provided $\inf \left(f_{x^{*}}-f\right)>\epsilon, f_{-} \leq x_{2}^{*} \leq f_{+}$, and $\|f\|_{C^{1,1}(\mathbf{R})} \leq c$, it holds that

$$
\left|G\left(x^{*}, z\right)-G^{\alpha}\left(x^{*}, z\right)\right|=|w(z)| \leq C\left\|K \phi_{x^{*}}^{\alpha}-g_{x^{*}}\right\|_{L^{2}\left(\Gamma^{x^{*}}\right)} \rightarrow 0
$$

as $\alpha \rightarrow 0$.

Up to this point the effect of noise in the measured data has been neglected. In practice we expect to measure $G_{\delta}(y, z)$ for $y \in \gamma^{*}$ rather than $G(y, z)$, with $\left\|G_{\delta}(\cdot, z)-G(\cdot, z)\right\|_{L^{2}\left(\gamma^{*}\right)}=$ $\delta>0$. Then we compute $G_{\delta}^{\alpha}\left(x^{*}, z\right)$, defined by $(18)$ with $G(y, z)$ replaced by the noisy data $G_{\delta}(y, z)$. From (18) and (19) and standard bounds for Tikhonov regularisation [18, Lemma 2.2.1] it follows that

$$
\begin{aligned}
\left|G\left(x^{*}, z\right)-G_{\delta}^{\alpha}\left(x^{*}, z\right)\right| & \leq C\left\|K \phi_{x^{*}}^{\alpha}-g_{x^{*}}\right\|_{L^{2}\left(\Gamma^{x^{*}}\right)}+\delta\left\|\phi_{x^{*}}^{\alpha}\right\|_{L^{2}\left(\gamma^{*}\right)} \\
& \leq C\left\|K \phi_{x^{*}}^{\alpha}-g_{x^{*}}\right\|_{L^{2}\left(\Gamma^{x^{*}}\right)}+\frac{\delta}{\sqrt{\alpha}}\left\|g_{x^{*}}\right\|_{L^{2}\left(\Gamma^{x^{*}}\right)} .
\end{aligned}
$$

In the usual case that the noise is a random variable, it follows from (20) that

$$
E\left(\left|G\left(x^{*}, z\right)-G_{\delta}^{\alpha}\left(x^{*}, z\right)\right|^{2}\right) \leq 2 C^{2}\left\|K \phi_{x^{*}}^{\alpha}-g_{x^{*}}\right\|_{L^{2}\left(\Gamma^{x^{*}}\right)}^{2}+2 \frac{\bar{\delta}^{2}}{\alpha}\left\|g_{x^{*}}\right\|_{L^{2}\left(\Gamma^{x^{*}}\right)}^{2},
$$

where $\bar{\delta}:=\sqrt{E\left(\delta^{2}\right)}$. Choosing $\alpha=a \bar{\delta}^{p}$, for some $a>0$ and $p \in(0,2)$, ensures that $E\left(\left|G\left(x^{*}, z\right)-G_{\delta}^{\alpha}\left(x^{*}, z\right)\right|^{2}\right) \rightarrow 0$ as $\bar{\delta} \rightarrow 0$.

\section{The Point Source Method in the Time Domain}

In the previous section we described an algorithm which reconstructs the total electric field up to the surface after the scattering of an incident field by a rough surface when the point source which gives rise to the incident field is time harmonic. In this section we consider the case when the point source emits a pulse. In particular we will have in mind the inverse rough surface scattering problem with a geometry and incident pulse similar to that arising in ground penetrating radar (GPR) applications. In GPR applications the radar waves are propagated in distinct pulses from a surface antenna, reflected off buried objects or features and detected back at the surface by a receiving antenna. From time of travel information the distance or depth of a feature can be calculated. General GPR systems use dipolar antennas ranging from $10 \mathrm{MHz}$ to $1000 \mathrm{MHz}$. Most of the antenna energy is concentrated around this one central frequency, but radar energy is produced in an interval that ranges up to one octave above and below the centre frequency.

For $F \in L^{1}(\mathbb{R})$ let $\hat{F} \in B C(\mathbb{R})$ denote the Fourier Transform of $F$, defined by

$$
\hat{F}(\omega)=\frac{1}{2 \pi} \int_{-\infty}^{\infty} F(t) e^{i \omega t} \mathrm{~d} t, \quad \omega \in \mathbb{R}
$$

If $F$ is sufficiently smooth (e.g. if $\left.F \in C^{2}(\mathbb{R}) \cap L^{1}(\mathbb{R})\right)$ then $\hat{F} \in L^{1}(\mathbb{R})$ and $F$ is given in terms of $\hat{F}$ by the inverse Fourier transform formula

$$
F(t)=\int_{-\infty}^{\infty} F(\omega) e^{-i \omega t} \mathrm{~d} \omega, \quad t \in \mathbb{R} .
$$


To model the pulse from a GPR antenna we assume that the incident field takes the form

$$
U^{i}(x, t)=\int_{-\infty}^{\infty} \frac{\Phi_{\omega / c}(x, z)}{\Phi_{\omega / c}\left(x_{0}, z\right)} \hat{F}^{i}(\omega) e^{-i \omega t} \mathrm{~d} \omega
$$

for some real-valued $F^{i} \in C^{2}(\mathbb{R})$ which is compactly supported, where $z$ is the source position, $x_{0} \neq z$ is some reference point where the incident field is measured and, for $x, y \in \mathbb{R}^{2}, x \neq y$,

$$
\Phi_{k}(x, y):=\frac{i}{4} H_{0}^{(1)}(k|x-y|), \quad k>0,
$$

while $\Phi_{k}(x, y):=\overline{\Phi_{-k}(x, y)}$, for $k<0$. It follows from (21) that, if $|x-z|=R:=\left|x_{0}-z\right|$, then

$$
U^{i}(x, t)=\int_{-\infty}^{\infty} \hat{F}^{i}(\omega) e^{-i \omega t} \mathrm{~d} \omega=F^{i}(t)
$$

so that $F^{i}(t)$ is the incident field at time $t$ at distance $R$ from the source (and in particular is the incident field at $\left.x_{0}\right)$.

The direct problem we consider in this section is as follows: Given $F^{i} \in C^{2}(\mathbb{R})$ which is compactly supported, find $U^{s} \in C^{2}(D \times \mathbb{R}) \cap C(\bar{D} \times \mathbb{R})$ such that

$$
\begin{aligned}
\Delta U^{s} & =\frac{1}{c^{2}} \frac{\partial^{2} U^{s}}{\partial t^{2}} & & \text { in } D \times \mathbb{R}, \\
U^{s} & =-U^{i} & & \text { in } \Gamma \times \mathbb{R}
\end{aligned}
$$

and, for some $t_{0} \in \mathbb{R}, U^{s}(\cdot, t)=0$ for $t<t_{0}$. By inspection, the solution to this direct problem is that the total field is given as

$$
U(x, t)=U^{i}(x, t)+U^{s}(x, t)=\int_{-\infty}^{\infty} \frac{G_{\omega / c}(x, z)}{\Phi_{\omega / c}\left(x_{0}, z\right)} \hat{F}^{i}(\omega) e^{-i \omega t} \mathrm{~d} \omega,
$$

where, for $k>0, G_{k}(x, z) e^{-i \omega t}$ denotes the total field corresponding to the time harmonic incident field $\Phi_{k}(x, z) e^{-i \omega t}$, so that $G_{k}(\cdot, z)$ is the solution to the Dirichlet boundary value problem in Section 2 and, for $k<0, G_{k}(\cdot, z):=\overline{G_{-k}(\cdot, z)}$.

A good approximation to an idealised pulse signature for a GPR antenna is given by

$$
F^{i}(t)=-e^{-\sigma\left(t+\frac{\epsilon}{\sqrt{\sigma}}\right)^{2}}+2 e^{-\sigma t^{2}}-e^{-\sigma\left(t-\frac{\epsilon}{\sqrt{\sigma}}\right)^{2}}, \quad t \in \mathbb{R},
$$

with $\sigma=25 \omega_{0}^{2} / 98, \omega_{0}=2 \pi f, f$ the nominal frequency, and $\epsilon=0.1$. The Fourier transform of $F^{i}$, given by (23), is

$$
\hat{F}^{i}(\omega)=-\sqrt{\frac{2 \pi}{3 \sigma}} e^{-\omega^{2} / 4 \sigma}\left(\cos \left(\frac{\omega \epsilon}{\sqrt{\sigma}}\right)-1\right) .
$$

In Figure 3 we plot $F^{i}(t)$ and $\left|\hat{F}^{i}(\omega)\right|^{2}$, given by (23) and (24) respectively, for nominal frequency $f=500 \mathrm{MHz}$.

To compute an approximation to $U^{s}(x, t)$ we proceed by first approximating $F^{i}(t)$ by a periodic function, $F_{T}^{i}(t)$, of some sufficiently large period $T$, defined by

$$
F_{T}^{i}(t)=F^{i}(t), \quad-T<t \leq T .
$$



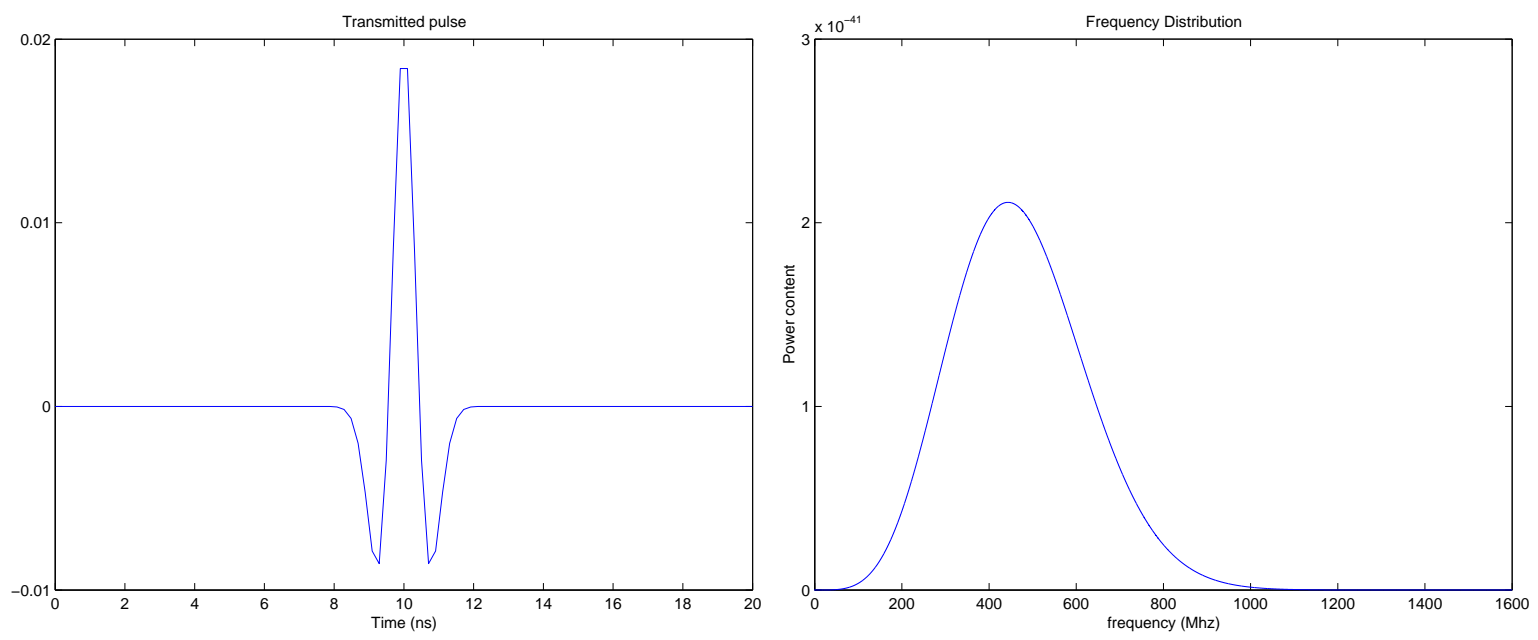

Figure 3: Incident pulse, $F^{i}(t)$, for nominal frequency $f=500 \mathrm{MHz}$, given by (23) (left hand plot) and the corresponding power spectrum, $\left|\hat{F}^{i}(\omega)\right|^{2}$, given by (24) (right hand plot).

We then sample $F_{T}^{i}(t)$ over a period of length $T$ at intervals $\Delta=\frac{T}{N}$ for some odd integer $N \in \mathbb{N}$, starting from some time $T_{1}$. Let $F_{p}^{i}:=F_{T}^{i}\left(T_{1}+\Delta p\right), p \in \mathbb{Z}$, denote the sampled values. Then, using the standard discrete Fourier transform formula [22],

$$
F_{p}^{i}=\sum_{j=0}^{N-1} X_{j} e^{-2 \pi i j p / N}, \quad p=0,1 \ldots, N-1,
$$

where

$$
X_{j}:=\frac{1}{N} \sum_{p=0}^{N-1} F_{p}^{i} e^{2 \pi i j p / N}, \quad j \in \mathbb{Z} .
$$

Now, since $F_{T}^{i}$ is periodic with period $T, F_{p+N}^{i}=F_{p}^{i}$ for $p \in \mathbb{Z}$. Hence, $X_{j+N}=X_{j}, j \in \mathbb{Z}$. Moreover, since we choose $N$ to be odd,

$$
X_{j}=\frac{1}{N} \sum_{p=-(N-1) / 2}^{(N-1) / 2} F_{p}^{i} e^{2 \pi i j p / N}, \quad j \in \mathbb{Z} .
$$

From this representation it becomes clear, since $F^{i}$ is real-valued, that $\bar{X}_{j}=X_{-j}, j \in \mathbb{Z}$. Since $X_{j+N}=X_{j}, j \in \mathbb{Z}$, it follows that

$$
F_{p}^{i}=\sum_{j=-(N-1) / 2}^{(N-1) / 2} X_{j} e^{-2 i \pi j \Delta p / T}, \quad p \in \mathbb{Z} .
$$

Let

$$
F_{N}^{i}(t):=\sum_{j=-(N-1) / 2}^{(N-1) / 2} X_{j} e^{-2 i \pi j\left(t-T_{1}\right) / T}, \quad t \in \mathbb{R} .
$$


Then $F_{N}^{i}(t+T)=F_{N}^{i}(t), t \in \mathbb{R}$, and

$$
F_{N}^{i}\left(T_{1}+\Delta p\right)=F_{p}^{i}=F_{T}^{i}\left(T_{1}+\Delta p\right), \quad p \in \mathbb{Z},
$$

so that $F_{N}^{i}$ interpolates $F_{T}^{i}$ at $T_{1}+\Delta p, p \in \mathbb{Z}$.

It is easy to see that $F_{N}^{i}(t)$ can be rewritten in the form

$$
F_{N}^{i}(t)=\frac{\alpha_{0}}{2}+\sum_{j=1}^{(N-1) / 2}\left[\alpha_{j} \cos \frac{2 \pi j\left(t-T_{1}\right)}{T}+\beta_{j} \sin \frac{2 \pi j\left(t-T_{1}\right)}{T}\right],
$$

with real coefficients $\alpha_{j}=2 \Re X_{j}$ and $\beta_{j}=2 \Im X_{j}$. In fact [17], there exists a unique function of this form (a so-called trigonometric polynomial of degree $(N-1) / 2$ and period $T$ ) which interpolates $F_{T}^{i}(t)$ at $T_{1}+\Delta p, p \in \mathbb{Z}$, and, if $F_{T}^{i} \in C^{n}(\mathbb{R})$, for some $n \in \mathbb{N}$, there holds the error estimate [17, Theorem 11.6]

$$
\left\|F_{N}^{i}-F_{T}^{i}\right\|_{\infty} \leq c \frac{\ln N}{N^{n}},
$$

for some constant $c>0$. We note that $F_{T}^{i} \in C^{n}(\mathbb{R})$ if $F^{i} \in C^{n}(\mathbb{R})$ and $T$ is large enough so that $(-T / 2, T / 2)$ contains the support of $F^{i}$.

With $F_{N}^{i}$ given by $(27)$ it holds that

$$
\hat{F}_{N}^{i}(\omega)=\sum_{j=-(N-1) / 2}^{(N-1) / 2} X_{j} e^{i \omega_{j} T_{1}} \delta\left(\omega-\omega_{j}\right),
$$

where $\omega_{j}=2 \pi j / T$ and $\delta$ is the Dirac delta function. We suppose that $X_{0}=0$ (the incident pulse has no DC component) which is the case for $F^{i}$ given by (23). Then, substituting in (21), an approximation to $U^{i}(x, t)$ is

$$
U_{N}^{i}(x, t):=\sum_{\substack{j=-(N-1) / 2 \\ j \neq 0}}^{(N-1) / 2} A_{j} \Phi_{k_{j}}(x, z) e^{-i \omega_{j} t}
$$

where $k_{j}:=\omega_{j} / c$ and

$$
A_{j}:=\frac{X_{j} e^{i \omega_{j} T_{1}}}{\Phi_{k_{j}}\left(x_{0}, z\right)}
$$

Since $\bar{A}_{j}=A_{j}$, we have that

$$
U_{N}^{i}(x, t)=2 \Re \sum_{j=1}^{(N-1) / 2} A_{j} \Phi_{k_{j}}(x, z) e^{-i \omega_{j} t} .
$$

Similarly, from (22), an approximation to the total field is

$$
U_{N}(x, t):=2 \Re \sum_{j=1}^{(N-1) / 2} A_{j} G_{k_{j}}(x, z) e^{-i \omega_{j} t} .
$$

For the direct scattering problem we are considering in this chapter the inverse problem analogous to that of Section 3 is the following one. Let $\gamma^{*}$ denote the finite horizontal line 
given by (10).

The Time Domain Inverse Problem. Given the source position $z \in D \backslash \gamma^{*}$, the incident field, $F^{i}(t)$, for $t \in \mathbb{R}$, at a reference point $x_{0} \neq z$, and measurements of the total field $U(x, t)$, for $x \in \gamma^{*}$ and $t \in \mathbb{R}$, determine $f$, i.e. the location of the infinite rough surface $\Gamma$.

To solve this inverse problem we will proceed analogously to Section 3 as follows. As a first step, compute, given $U(x, t)$, for $x \in \gamma^{*}, t \in \mathbb{R}$, an approximation to $U(x, t)$ for $x \in D, t \in \mathbb{R}$. As a second step determine $\Gamma$ as the curve on which the approximation to $U(x, t)$ is minimised.

To construct an approximation to $U(x, t)$ for $x \in D, t \in \mathbb{R}$, we propose, as a first stage, to compute the constants $X_{j}$ and $A_{j}$, given by (25) and (28), and approximate $F^{i}$ by $F_{N}^{i}$, given by (27). The corresponding total field is given by (29). As a second stage we propose to, similarly, approximate the measured data by $\tilde{U}_{N}(x, t)$, for $x \in \gamma^{*}, t \in \mathbb{R}$, where $\tilde{U}_{N}(x, t)$ is the $T$-periodic trigonometric polynomial of degree $\leq(N-1) / 2$ which interpolates $U(x, \cdot)$ at $T_{1}+\Delta p, p=0,1, \ldots, N-1$. This approximation takes the form

$$
\tilde{U}_{N}(x, t)=2 \Re \sum_{j=1}^{(N-1) / 2} a_{j}(x) e^{-i \omega_{j} t},
$$

for some functions $a_{j}(x)$, determined by the interpolation requirement. Comparing (29) with (30) we obtain, approximately, values for $G_{k_{j}}(x, z)$ for $j=1, \ldots,(N-1) / 2$ and $x \in \gamma^{*}$, namely $G_{k_{j}}(x, z)=a_{j}(x) / A_{j}$. Given this frequency domain data on $\gamma^{*}$ we can compute the point source method approximations $G_{k_{j}}^{\alpha}(x, z)$, given by (18). Then, as the third and final stage, we can approximate the total field by $(29)$ with $G_{k_{j}}(x, z)$ replaced by $G_{k_{j}}^{\alpha}(x, z)$, giving the approximation

$$
U_{N}^{\alpha}(x, t):=2 \Re \sum_{j=1}^{(N-1) / 2} A_{j} G_{k_{j}}^{\alpha}(x, z) e^{-i \omega_{j} t}, \quad x \in \mathbb{R}^{2} \backslash\left(\gamma^{*} \cup\{z\}\right), t \in \mathbb{R},
$$

where we denote $G^{\alpha}\left(x^{*}, z\right)$ by $G_{k_{j}}^{\alpha}\left(x^{*}, z\right)$ to indicate its dependence on $k_{j}$. We will call this a time domain point source method approximation for the total field $U(x, t)$.

Given that $U(x, t)=0$ for $x \in \Gamma, t \in \mathbb{R}$, we propose to locate the surface $\Gamma$ as the minimum of

$$
P(x)=\frac{1}{T} \int_{0}^{T}\left(U_{N}^{\alpha}(x, t)\right)^{2} \mathrm{~d} t .
$$

By Parseval's theorem,

$$
P(x)=2 \sum_{j=1}^{(N-1) / 2}\left|A_{j}\right|^{2}\left|G_{k_{j}}^{\alpha}(x, z)\right|^{2} .
$$

\section{$5 \quad$ Numerical Results}

Throughout the numerical examples we assume a wave speed $c=c_{0} / \sqrt{10}$, where $c_{0}$ is the velocity of light in vacuo. This wave speed is appropriate for radar waves in dry sandy coastal land [14], and gives a wavelength of $\lambda=c / f=0.19 \mathrm{~m}$ when the frequency is $f=500 \mathrm{MHz}$. We 
assume that $F^{i}$ is given by (23) with $f=500 \mathrm{MHz}$, so that the incident pulse is representative of a GPR antenna with nominal frequency $500 \mathrm{MHz}$. The measurement line in the inverse problem is $\gamma^{*}=\left\{\left(x_{1}, H\right)|| x_{1} \mid \leq 10 \lambda=1.9 \mathrm{~m}\right\}$ and we fix the height of the measurement line as $H=4 \lambda=0.76 \mathrm{~m}$. The source of the incident field is at $z=(0,4 \lambda)=(0,0.76) \mathrm{m}$, in the centre of the measurement line. The scattering surface $\Gamma$ is the graph of the function

$$
f\left(x_{1}\right)=\frac{11 \lambda}{8}+\frac{\lambda}{8} \cos \left(\frac{2 x_{1}}{\lambda}\right)+\frac{\lambda}{4} \sin \left(\frac{2 x_{1}}{3 \lambda}\right), \quad x_{1} \in \mathbf{R},
$$

for which (2) holds with $f_{-}=\lambda, f_{+}=7 \lambda / 4$.

We calculate $U_{N}^{\alpha}\left(x^{*}, t\right)$ for $N=49$, using $(31)$, for $0<t \leq T=20 \mathrm{~ns}$, and for $\left|x_{1}^{*}\right| \leq 5 \lambda$ and $\lambda / 2 \leq x_{2}^{*} \leq 7 \lambda / 2$. $G_{k_{j}}^{\alpha}\left(x^{*}, z\right)$, where $k_{j}=2 \pi f_{j} / c$ with $f_{j}=50 j \mathrm{MHz}$, is calculated using the point source method described in Section 3, for $j=1, \ldots, 24$ and for $x^{*}$ on a grid with 9.6 points per wavelength $(\lambda)$ in both coordinate directions. For each $x^{*}=\left(x_{1}^{*}, x_{2}^{*}\right)$ we make the choice $\Gamma^{x^{*}}=\left\{\left(x_{1}, f_{x^{*}}\left(x_{1}\right)\right) \mid x_{1} \in \mathbb{R}\right\}$, with $f_{x^{*}}\left(x_{1}\right)=-\frac{3 \lambda}{4} \exp \left(-\left(\frac{x_{1}-x_{1}^{*}}{8 \lambda}\right)^{2}\right)+\frac{\lambda}{4}+x_{2}^{*}$. We choose $h^{*}=x_{2}^{*}-d$, with $d=f_{+}-f_{-}+\lambda / 4=\lambda$.

In the numerical implementation we approximate the integral in (12) by the trapezium rule with step length $h=\lambda / 10$ and collocate at equally spaced points $x$, with the same spacing $h$, only on the part of $\Gamma^{x^{*}},\left\{x \in \Gamma^{x^{*}}|| x_{1} \mid \leq 19 \lambda\right\}$. Numerical experiments suggest that, for this particular configuration, the accuracy of reconstructions in the region below $\gamma^{*}$ is not significantly improved by collocating on a larger part of $\Gamma^{x^{*}}$. This then leads to an approximation of (12) as a linear system, with coefficient matrix $\mathbf{K}$. We regularise this linear system using Tikhonov regularisation with regularisation parameter $\alpha=10^{-4}$, unless otherwise stated, forming the discrete analogue of (14), replacing $K$ and $K^{*}$ by $\mathbf{K}$ and $\mathbf{K}^{*}$. Note that the "measured" values of $G_{k_{j}}(x, z)$ are computed by the boundary integral equation method and super-algebraically convergent Nyström method proposed in [21], with horizontal spacing between quadrature points of $\lambda / 10$, and truncating the boundary integral so that $\Gamma$ is replaced by $\left\{x \in \Gamma|| x_{1} \mid \leq 100 \lambda\right\}$ (see $[21,20]$ for further details).

To compute the noisy reconstructed total field we calculate $U_{N, \delta}^{\alpha}\left(x^{*}, t\right)$ as for $U_{N}^{\alpha}\left(x^{*}, t\right)$ but with noise added to each measured value of $G_{k_{j}}(x, z), x \in \gamma^{*}$, by replacing $G_{k_{j}}(x, z)$ by $G_{k_{j}, \delta}(x, z)=G_{k_{j}}(x, z)+N(x)$, where $N(x)$ and $N(y)$ are independently distributed for $x \neq y$, and where, for each $x$, the real and imaginary parts of $N(x)$ are independently and identically normally distributed with mean 0 and standard deviation chosen such that $\sqrt{E\left(|N(x)|^{2}\right)}=\frac{\delta^{*}}{100}\left|\Phi_{k_{j} j}(x, z)\right|$, where $\delta^{*}$ is the specified percentage error. Note that this implies that $\delta^{*}$ is related to $\delta=\|N\|_{L^{2}\left(\gamma^{*}\right)}$ by

$$
\bar{\delta}:=\sqrt{E\left(\delta^{2}\right)}=\frac{\delta^{*}}{100}\left\|\Phi_{k_{j}}(\cdot, z)\right\|_{L^{2}\left(\gamma^{*}\right)} .
$$

Figures 4 and 5 show snapshots of the reconstructed total field $U_{N, \delta}^{\alpha}\left(x^{*}, t\right)$ at a sequence of times for the above geometry, with $\delta^{*}=0$ (no measurement noise) and $\delta^{*}=5$ (5\% error) in figures 4 and 5 , respectively. Also shown in the figures is the scattering surface $\Gamma$, the position of which is unknown, of course, to the reconstruction algorithm. In Figure 4, with no measurement noise, a clear incident pulse is visible as is the pulse reflected from the surface. In fact the reconstructed total field above $\Gamma$ is very accurate, as shown by the results in Figure 6 discussed below, and suggested by Theorem 3.1, though note that Theorem 3.1 only applies when $\Gamma^{x^{*}}$ lies above $\Gamma$, and so certainly does not apply when $x_{2}^{*}<f\left(x_{1}^{*}\right)+\lambda / 2$. Since the position of $\Gamma$ is unknown, we are computing $U_{N, \delta}^{\alpha}\left(x^{*}, t\right)$ on a grid which includes points above 
and below $\Gamma$. We have no theory for what we are computing when $x^{*}$ is below $\Gamma$, but perhaps it is an approximation to the solution, $U\left(x^{*}, t\right)$, to the wave equation extended across the boundary $\Gamma$. It appears to us that some sort of image of the incident field, reflected in $\Gamma$, can be seen in Figure 7 leaving an approximate image source position at time $t=0$ and rising up to interfere destructively with the incident field to satisfy the boundary condition that $U=0$ on $\Gamma$. Comparing Figures 4 and 5 it is clear that adding $5 \%$ random error to the measurements has a significant effect on the reconstruction of the total field achieved. However, despite the effects of this noise, the incident and scattered waves can be clearly distinguished in Figure 5 .

In Figures 6 and 7 we take a more detailed look at the quality of the reconstructions, comparing the reconstructed total field $U_{N, \delta}^{\alpha}(x, t)$, for $0 \leq t \leq T=20 \mathrm{~ns}$, with $U_{N}(x, t)$ given by (29), at several points $x$ above $\Gamma$, for the same geometry and noise levels as in Figures 4 and 5 , respectively. In the two Figures 6 and 7 the incident and reflected pulse can be seen in the top two plots (the reflected pulse with phase change of $180^{\circ}$, appropriate to the boundary condition that the total field vanishes). The incident and reflected pulses begin to merge in the 3 rd plot, for $x=(0,1.6 \lambda)$ (the surface passes through $(0,1.5 \lambda)$ ).

The reconstructed total fields agree very accurately with the exact values of the total field in Figure 6. When 5\% noise is added to the data (Figure 7), even though we can see errors in the reconstructed total fields, the incident and reflected pulses are clearly visible and correctly positioned on the time axis.

Although these reconstructions are for the case when the total electric field vanishes on the surface, the same formula for reconstructing the total field from measured data can be expected to work regardless of the boundary condition. There would appear, in fact, to be good prospects for locating the surface, even without knowledge of the boundary condition, as the locus of those points where the incident and reflected pulses coincide in time.

In Figure 8 we predict the surface location as the minimum of $P(x)$, given by (32) and (33). We first interpolate $P(x)$, calculated for $\left|x_{1}\right| \leq 5 \lambda$ and $1 / 2 \lambda \leq x_{2} \leq 3 / 2 \lambda$ at 9.6 points per wavelength $(\lambda)$, to 19 points per wavelength, and this is what is shown in the left hand side of Figure 8. On the right hand side we put a grid over the same region and, in each column, colour in black the square in which $P(x)$ is minimised as a function of $x_{2}$. The squares coloured in black are thus our reconstruction of the position of the scattering surface $\Gamma$. It can be seen that, for this particular configuration, the reconstruction is near perfect if no noise is added to the measurements, with errors a small fraction of the nominal wavelength $\lambda$. When $5 \%$ noise is added the quality of the reconstruction is almost as good for $-2 \lambda \leq x_{1} \leq 3.5 \lambda$, but effectively no useful reconstruction is obtained for $\left|x_{1}\right| \geq 4 \lambda$. Bearing in mind that the source is in the centre of the finite measurement line which is $20 \lambda$ in length, our speculation is that, on a geometrical optics basis, no significant proportion of the incident wave power is being scattered through the finite measurement line from parts of the surface with $\left|x_{1}\right|>5 \lambda$ so that effectively no information is being collected about the parts of the surface with $\left|x_{1}\right|>5 \lambda$.

Finally, we investigate in more detail the effect of the noise level on the accuracy of predicting the location of the surface. For the surface (34), we compute $U_{N, \delta}^{\alpha}(x, z)$ on the two vertical lines, $x_{1}=0$ and $x_{1}=4 \lambda$, for $\lambda / 2<x_{2}<5 \lambda / 2$. On each vertical line we compute the value of $x_{2}$, denoted by $X_{2}$, which minimises $P(x)$, given by (33), over some range of $x_{2}$. The range of $x_{2}$ is either $\lambda / 2<x_{2}<5 \lambda / 2$ or the part of this interval which lies within distance $\lambda / 3$ of the surface, i.e. $\max \left(\lambda / 2, f\left(x_{1}\right)-\frac{\lambda}{3}\right)<x_{2}<\min \left(f\left(x_{1}\right)+\frac{\lambda}{3}, 5 \lambda / 2\right) . X_{2}$ is thus a time domain point source estimate of the surface height, $f\left(x_{1}\right)$. Using each of the ranges of $x_{2}, X_{2}$ is computed for 20 values of the percentage error, $\delta^{*}$, logarithmically spaced on the interval $0.0001<\delta^{*}<12$. In these calculations we choose the regularisation parameter to 
be $\alpha=10^{-4} \delta^{*} / 5$. Then $\alpha \rightarrow 0$ as $\delta^{*} \rightarrow 0$, and the theory of Section 3, coupled with (35), predicts that, for each wavenumber $k_{j}, j=1, \ldots, 24$, the point source estimate $G_{\delta}^{\alpha}\left(x^{*}, z\right)$ of $G\left(x^{*}, z\right)$, the main approximation in our reconstruction algorithm, satisfies, if $\Gamma^{x^{*}}$ lies above $\Gamma$

$E\left(\left|G\left(x^{*}, z\right)-G_{\delta}^{\alpha}\left(x^{*}, z\right)\right|^{2}\right) \leq 2 C^{2}\left\|K \phi_{x^{*}}^{\alpha}-g_{x^{*}}\right\|_{L^{2}\left(\Gamma^{x^{*}}\right)}^{2}+10 \delta^{*}\|\Phi(\cdot, z)\|_{L^{2}\left(\gamma^{*}\right)}^{2}\left\|g_{x^{*}}\right\|_{L^{2}\left(\Gamma^{x^{*}}\right)}^{2} \rightarrow 0$

as $\delta^{*} \rightarrow 0$.

In Figure 9 we plot $\left|X_{2}-f\left(x_{1}\right)\right| / \lambda$ against $\delta^{*}$, i.e. we plot the distance of the predicted surface location from the actual surface (in wavelengths) as the noise in the measurement data increases. In Figure 9 the left hand plot shows the results for $x_{1}=0$ (so $f\left(x_{1}\right)=1.5 \lambda$ ) and the right hand plot shows the results for $x_{1}=4 \lambda$ (so $\left.f\left(x_{1}\right)=1.4711 \lambda\right)$. In each graph the dashed line indicates the distance of $X_{2}$ from $f\left(x_{1}\right)$ when $x_{2}$ minimises $P(x)$ over the range $\lambda / 2<x_{2}<5 \lambda / 2$, while the solid line shows the distance of $X_{2}$ from $f\left(x_{1}\right)$ when $x_{2}$ minimises $P(x)$ over the range $f\left(x_{1}\right)-\lambda / 3<x_{2}<f\left(x_{1}\right)+\lambda / 3$. The dotted line is the graph of $C\left(\delta^{*}\right)^{1 / 2}$, for some constant $C>0$.

As expected, as the percentage of noise added to the measurement data increases, the general trend is that the accuracy with which the surface location is predicted worsens. The large fluctuations about this general trend are mainly due to the random nature of the noise. (Note that $\delta^{*}$ is proportional to the standard deviation of the population from which the random noise is sampled rather than being the root mean square of the particular realisation of the random noise.) As noted already in connection with Figure 8, the accuracy of prediction is much worse for $x_{1}=4 \lambda$ than for $x_{1}=0$. Precisely, in Figure 9, the surface location is predicted to within $10^{-3} \lambda$ for $x_{1}=0$ and to within $10^{-1.4} \lambda \approx 0.04 \lambda$ for $x_{1}=4 \lambda$, for small values of $\delta^{*}$, the percentage error.

At $x_{1}=0,\left|X_{2}-f\left(x_{1}\right)\right|$ appears to approach zero approximately in proportion to $\left(\delta^{*}\right)^{1 / 2}$; the rate of convergence at $x_{1}=4 \lambda$ is possibly a little slower. In [18, 19] similar graphs are plotted for the corresponding frequency domain scattering problem with $F^{i}$ time harmonic with wavelength $\lambda$. In the frequency domain case (solving the inverse problem of Section 3) similar rates of convergence as $\delta^{*} \rightarrow 0$ are observed. However the accuracy of the predicted surface location is improved significantly when using the time domain algorithm, especially for larger $\delta^{*}$.

The plotting of the two curves in Figure 9 is motivated by Remark 3.1 which suggests that there may be issues of non-uniqueness for the inverse problem. Precisely, for the case of an incident time harmonic plane wave, with wavelength $\lambda$, it is pointed out in the remark that two flat boundaries a distance $\lambda / 2$ apart can produce the same scattered field. The dashed curves in Figure 9 suggest that, if the search for the surface is constrained, by a priori knowledge, to a neighbourhood of the actual surface position, the reconstructions are improved for larger values of the percentage error, $\delta^{*}$. Precisely, a significant improvement is seen when $\delta^{*}>8$ for $x_{1}=0$ and when $\delta^{*}>2$ for $x_{1}=4 \lambda$.

\section{Conclusions}

In this paper we have proposed point source method algorithms for inverse scattering by unbounded rough surfaces. These algorithms, in the first instance, reconstruct the total field above the scattering surface from limited measurement, on a finite line above the surface. For the case of a time harmonic incident field we have briefly sketched a theoretical justification for 
our method, culminating in the error bound of Theorem 3.1 - for a more complete justification see $[18,19]$. Based on this frequency domain algorithm we have proposed a time domain point source method, in which we approximate the actual incident field by a finite sum of time harmonic fields and then apply the point source algorithm for time harmonic waves. A rigorous convergence analysis has not been established for this method (it seems to us to need an understanding of how the constant $C$ in Theorem 3.1 depends on the wavenumber). But numerical results show that the time domain point source method can be very effective.

In the numerical experiments that we have carried out we have chosen an incident pulse that is similar to that arising in ground penetrating radar applications. With the measurement line $2 \frac{1}{4}$ wavelengths from the mean surface level, the reconstructed total fields agree very well with the exact total field, and when $5 \%$ noise is added to the measurements the incident and reflected pulse positions in time are very accurate and clearly observable. Having reconstructed the total field, the scattering surface, on which the total field vanishes, is located as the locus of minima of the reconstructed total field. These reconstructions are accurate, over a large part of the scattering surface, with the surface height recovered to within a small fraction of a wavelength, even with $5 \%$ noise. We have also investigated numerically the convergence of the reconstructed surface to the true surface as the noise level (and the regularisation parameter in the method) tend to zero. A convergence rate of approximately $\left(\delta^{*}\right)^{1 / 2}$, where $\delta^{*}$ is the percentage error, is observed.

Finally we remark that, although all the reconstructions in this paper have been for the case where the total field vanishes on the boundary, it seems to us that the time domain point source method is likely to have more general application. In particular, the formulae for reconstructing the total field up to the surface as a function of time can be expected to work for general boundary conditions. It seems to us that there are good prospects then for identifying the surface, even without knowledge of the boundary condition, as the locus of those points where the incident and reflected pulses coincide in time.

\section{References}

[1] T. Arens. Why linear sampling works! Inverse Problems, 20:163-173, 2004.

[2] T. Arens and Kirsch. A. the factorization method in inverse scattering from periodic structures. Inverse Problems, 19:1195-1211, 2003.

[3] S. N. Chandler-Wilde. The impedance boundary value problem for the Helmholtz equation in a half-plane. Math. Meth. Appl. Sci., 20:813-840, 1997.

[4] S. N. Chandler-Wilde and D. C. Hothersall. Efficient calculation of the Green function for acoustic propagation above a homogeneous impedance plane. J. Sound Vib., 180:705-724, 1995.

[5] S. N. Chandler-Wilde and C. R. Ross. Scattering by rough surfaces: the Dirichlet problem for the Helmholtz equation in a non-locally perturbed half-plane. Math. Meth. Appl. Sci., 19:959-976, 1996.

[6] S. N. Chandler-Wilde, C. R. Ross, and B. Zhang. Scattering by rough surfaces. In J. DeSanto, editor, Proceedings of the Fourth International Conference on Mathematical and Numerical Aspects of Wave Propagation, pages 164-168. SIAM, 1998. 
[7] S. N. Chandler-Wilde, C. R. Ross, and B. Zhang. Scattering by infinite one-dimensional rough surfaces. Proc. R. Soc. Lon. A, 455:3767-3787, 1999.

[8] M. Cheney. A mathematical tutorial on synthetic aperture radar. SIAM Rev, 43:301-312, 2001.

[9] R. Coifman, M. Goldberg, T. Hrycak, M. Israeli, and V. Rokhlin. An improved operator expansion algorithm for direct and inverse scattering computations. Waves in Random Media, 9:441-457, 1999.

[10] D. Colton, J. Coyle, and P. Monk. Recent developments in inverse acoustic scattering theory. SIAM Rev., 42:369-414, 2000.

[11] D. Colton and A. Kirsch. A simple method for solving inverse scattering problems in the resonance region. Inverse Problems, 12:383-393, 1996.

[12] J. A. DeSanto and R. J. Wombell. The reconstruction of shallow rough-surface profiles from scattered field data. Inverse Problems, 7:L7-L12, 1991.

[13] J. Elschner and M. Yamamoto. An inverse problem in periodic diffractive optics: Reconstruction of lipschitz grating profiles. Applicable Analysis, 81:1307-1328, 2002.

[14] D. Goodman and L. Conyers. Ground Penetrating Radar, An Introduction for Archaeologists. AltaMira, 1997.

[15] M. Ikehata. On reconstruction in the inverse conductivity problem with one measurement. Inverse Problems, 16:785-793, 2000.

[16] A. Kirsch. Characterization of the shape of a scattering obstacle using the spectral data of the far field operator. Inverse Problems, 14:1489-1512, 1998.

[17] R. Kress. Linear Integral Equations. Springer, Berlin, 1989.

[18] C. D. Lines. Inverse Scattering by Unbounded Rough Surfaces. PhD thesis, Brunel University, 2003.

[19] C. D. Lines and S. N. Chandler-Wilde. A point source method for inverse scattering by rough surfaces. In preparation.

[20] A. Meier. Numerical Treatment of Integral Equations on the Real Line with Application to Acoustic Scattering by Unbounded Rough Surfaces. PhD thesis, Brunel University, 2001.

[21] A. Meier, T. Arens, S. N. Chandler-Wilde, and A. Kirsch. A Nyström method for a class of integral equations on the real line with applications to scattering by diffraction gratings and rough surfaces. J. Int Equ. Appl., 12:281-321, 2000.

[22] D. E. Newland. An Introduction to Random Vibrations, Spectral and Wavelet Analysis. Longman, 1993.

[23] R. Potthast. A point source method for inverse acoustic and electromagnetic obstacle scattering problems. IMA J. Appl. Math., 61:119-140, 1998. 
[24] R. Potthast. Point Sources and Multipoles in Inverse Scattering Theory. CRC Press, 2001.

[25] R. Potthast and D. Luke. The no response test - a sampling method for inverse scattering problems. Siam J. Appl. Math., 63:1292-1312, 2003.

[26] R. Potthast, J. Sylvester, and S. Kusiak. A 'Range test' for determining scatterers with unknown physical properties. Inverse Problems, 19:533-547, 2003.

[27] C. Sheppard. Imaging of random surfaces and inverse scattering in the Kirchhoff approximation. Waves in Random Media, 8:53-66, 1998.

[28] M. Spivack. Direct solution of the inverse problem for rough scattering at grazing incidence. J. Phys. A: Math. Gen, 25:3295-3302, 1992.

[29] R. J. Wombell and J. A. DeSanto. Reconstruction of rough-surface profiles with the Kirchhoff approximation. J. Opt. Soc. Am., 8:1892-1897, 1991.

[30] C. Ying and A. Noguchi. Rough surface inverse scattering problem with gaussian beam illumination. IEICE Trans. Electron., E77-C(11):1781-1785, 1994. 

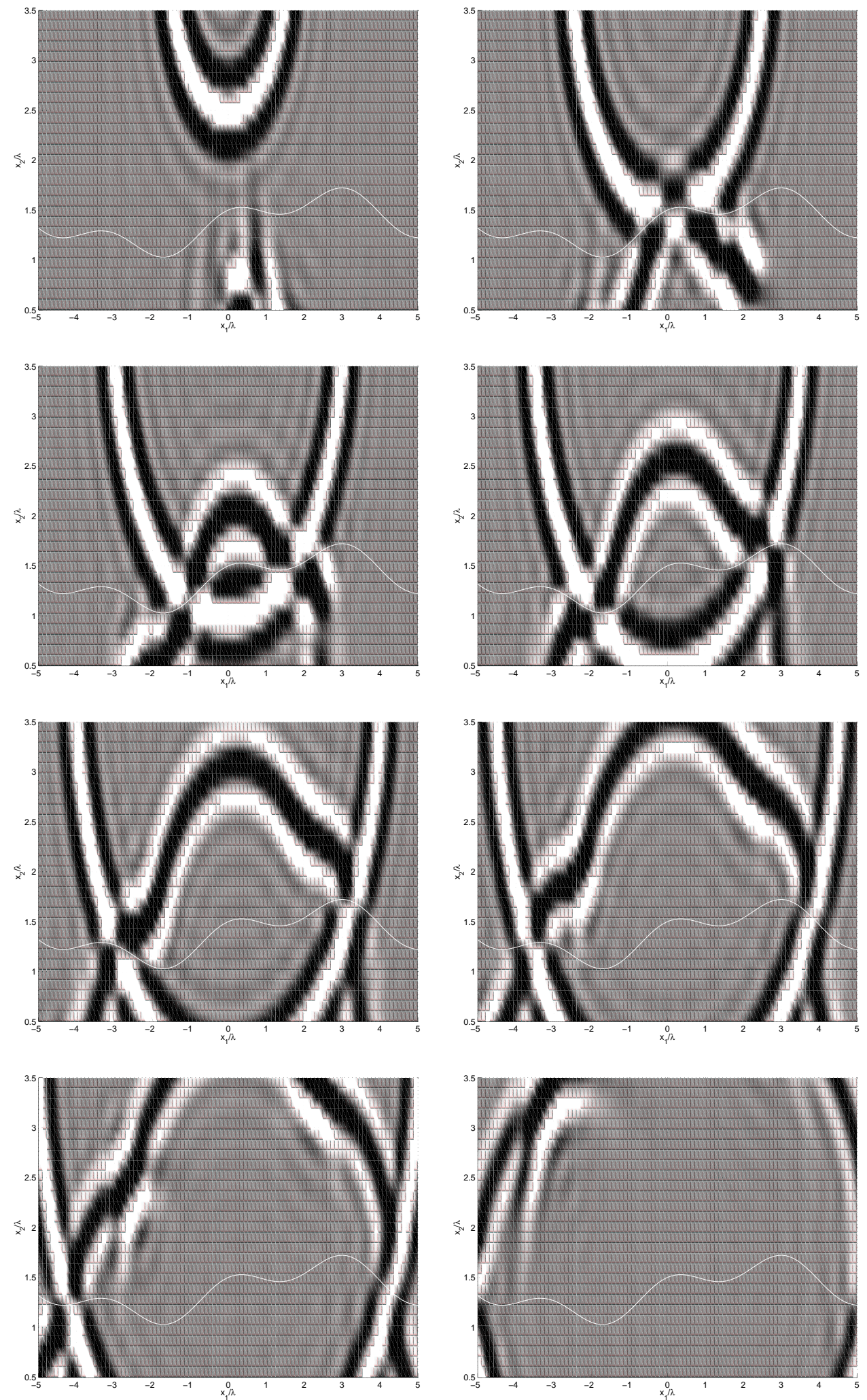

Figure 4: The reconstructed total field, $U_{N}^{\alpha}(x, t)$, for $t=3.2 \mathrm{~ns}, 5.2 \mathrm{~ns}, 6.2 \mathrm{~ns}, 7.2 \mathrm{~ns}, 8.2 \mathrm{~ns}$, $9.2 \mathrm{~ns}, 10.2 \mathrm{~ns}$ and $12.2 \mathrm{~ns}$ (from top left to bottom right) for surface (34). 

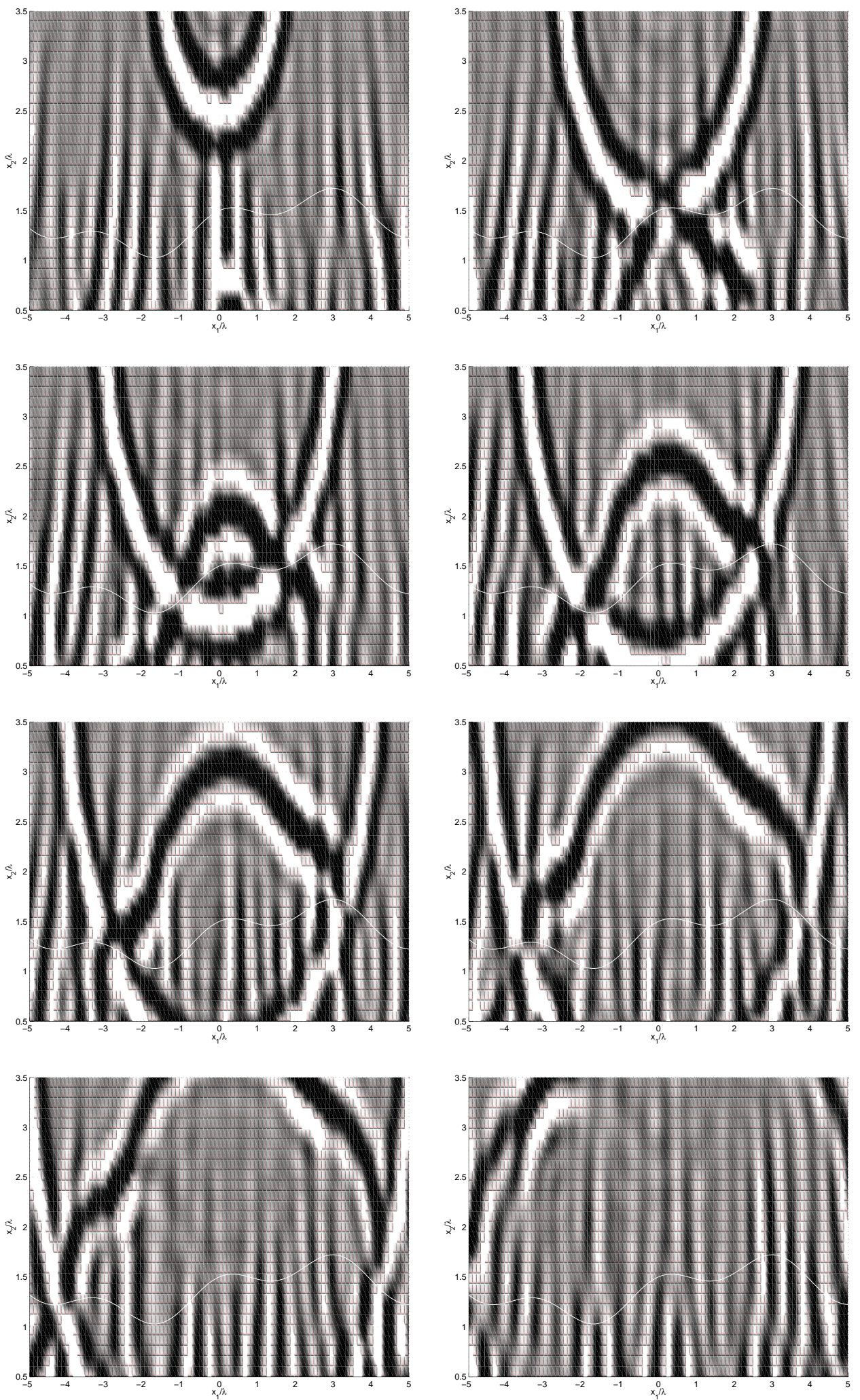

Figure 5: The reconstructed total field, $U_{N, \delta}^{\alpha}(x, t)$, for $t=3.2 \mathrm{~ns}, 5.2 \mathrm{~ns}, 6.2 \mathrm{~ns}, 7.2 \mathrm{~ns}, 8.2 \mathrm{~ns}$, $9.2 \mathrm{~ns}, 10.2 \mathrm{~ns}$ and $12.2 \mathrm{~ns}$ (from top left to bottom right) for surface $(34)$ with $\delta^{*}=5$, i.e $5 \%$ noise added to the measurement data. 

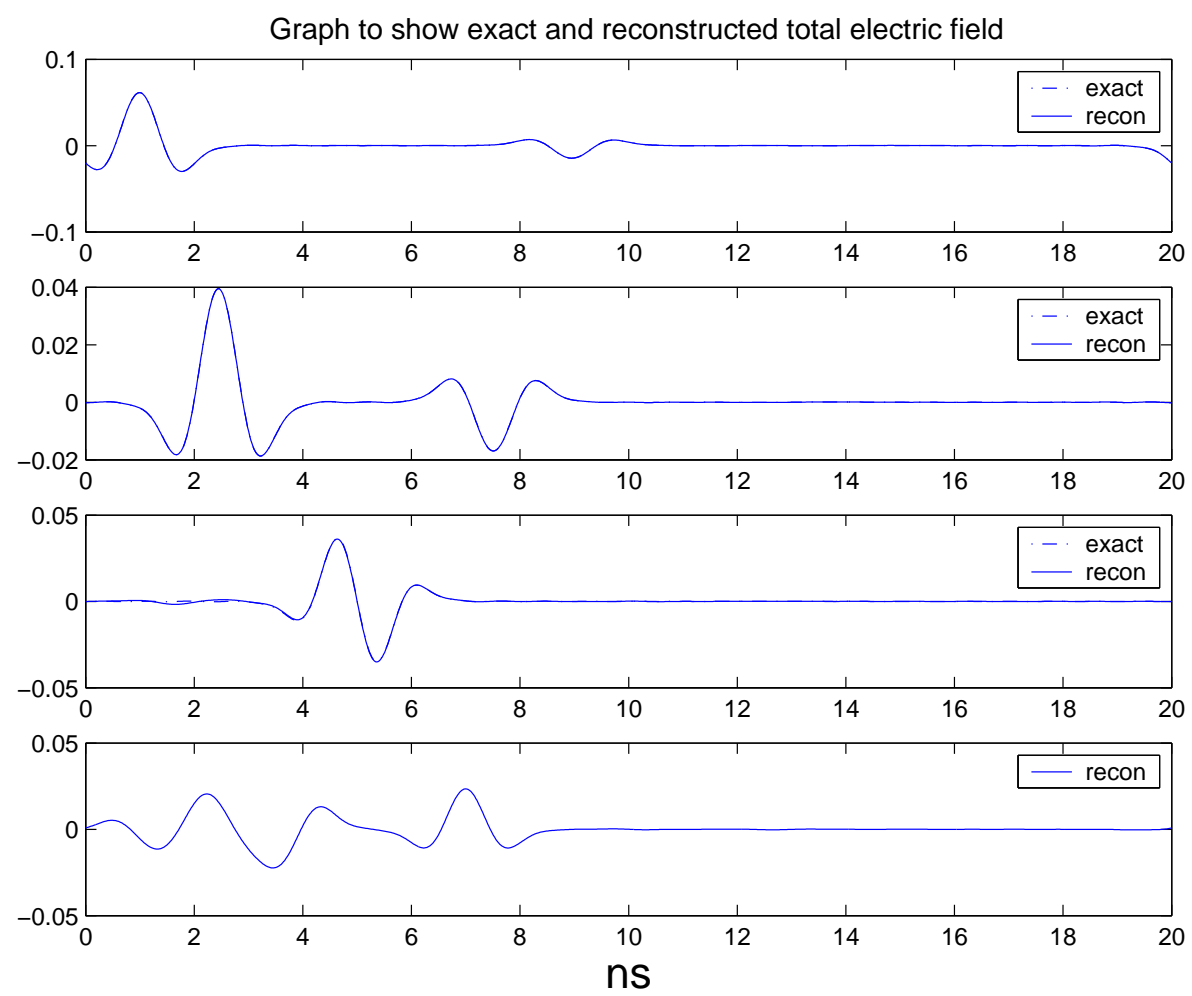

Figure 6: Comparison of the reconstructed total field, $U_{N}^{\alpha}(x, t)$, and the exact total field, $U_{N}(x, t)$, for the surface (34) on the time interval $0<t \leq T=20 \mathrm{~ns}$. The total fields are compared at the points $x=(0,3.5 \lambda), x=(0,2.6 \lambda), x=(0,1.6 \lambda)$ and $x=(0,0.5 \lambda)$ (from top to bottom). Note that the surface is at height $1.5 \lambda$. Thus the bottom plot shows a reconstructed total field for a point $x$ below the surface. The reconstructions are so accurate that most of the graphs are superposed. 

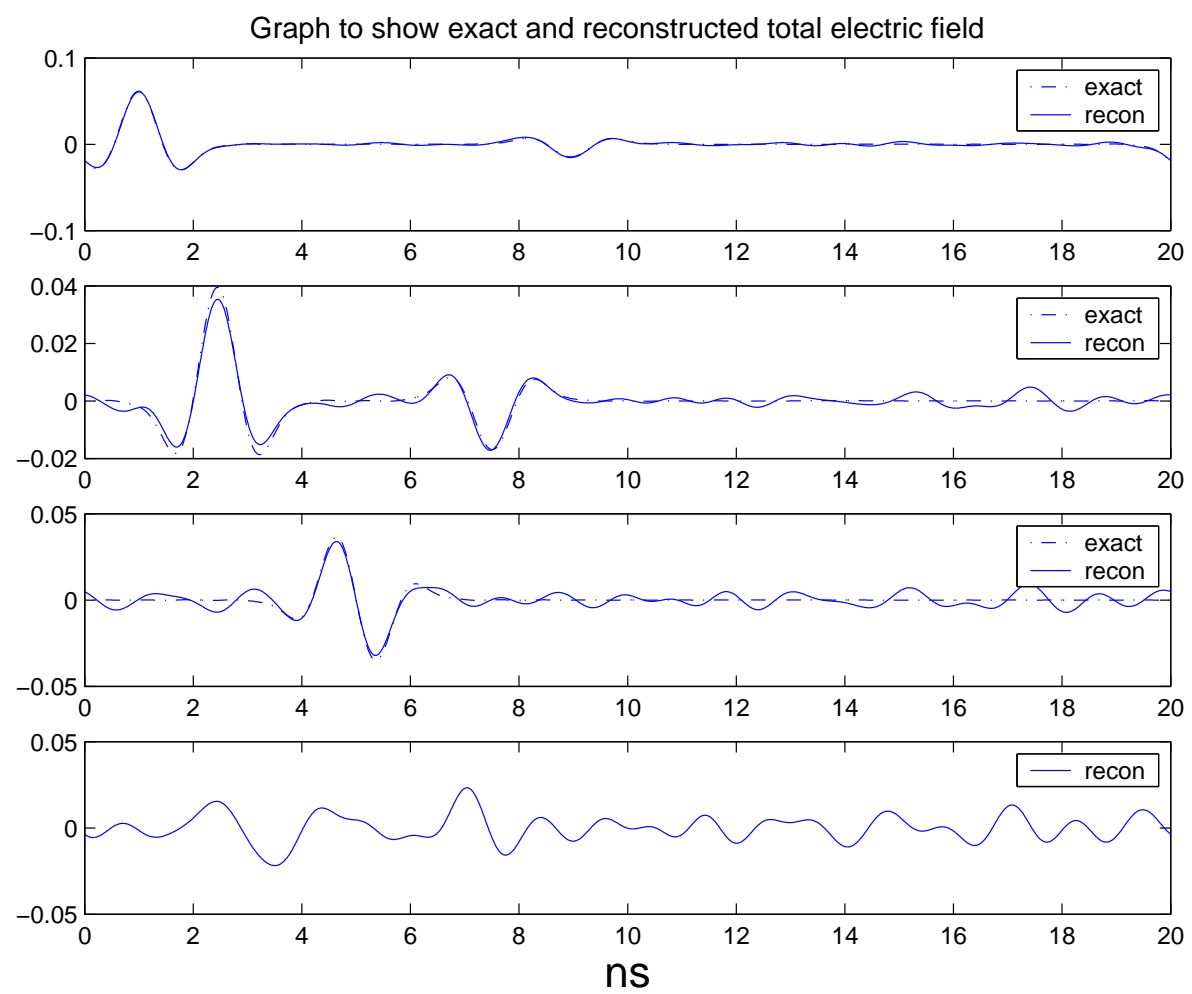

Figure 7: As Figure 6 but with $5 \%$ noise added to the measurements. 

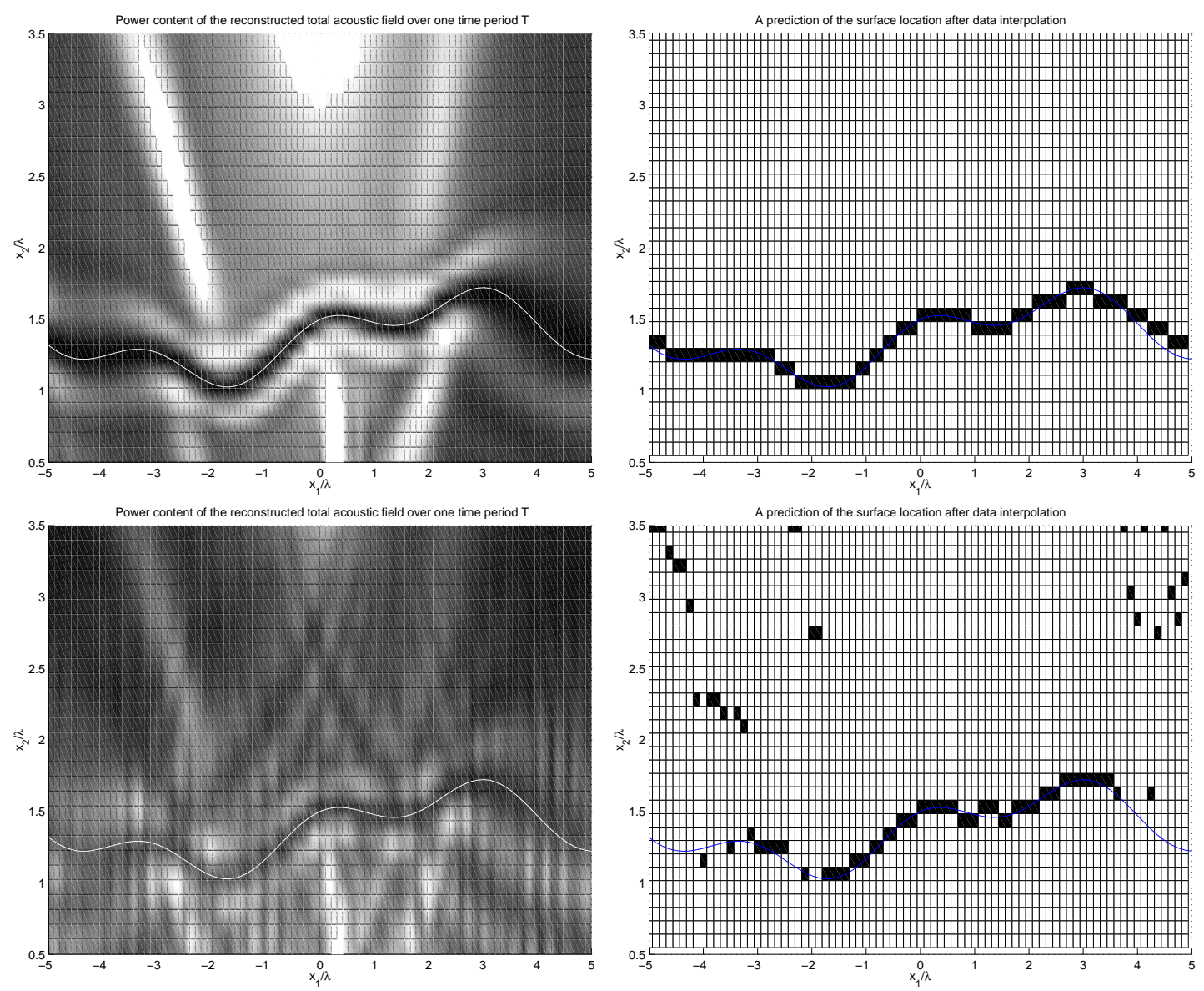

Figure 8: A plot of the power content, $P(x)$, of the total electric field (left hand side). On the right hand side we predict the surface location from this plot by colouring in, in each column, the square in which $P(x)$ is minimised as a function of $x_{2}$. The bottom two plots show the case when $5 \%$ noise is added to the measurements. 

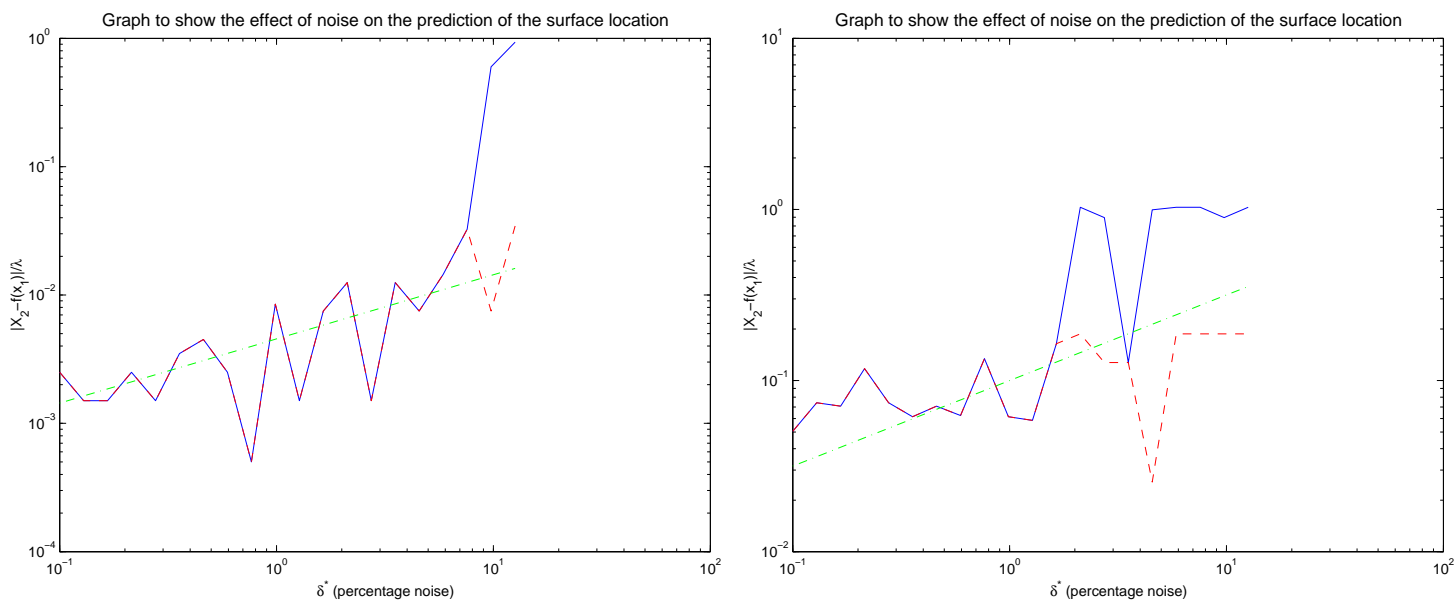

Figure 9: Results showing the accuracy of the predicted surface location, $\left|X_{2}-f\left(x_{1}\right)\right| / \lambda$, as $\delta^{*}$ increases. Shown are results for the rough surface (34) where $U_{N, \delta}^{\alpha}(x, z)$ is calculated on the vertical line $x_{1}=0$ (left hand plot) and on the vertical line $x_{1}=4 \lambda$ (right hand plot). The dashed line shows the distance of $X_{2}$ from $f\left(x_{1}\right)$ when $x_{2}$ minimises $P(x)$ over the range $\lambda / 2<x_{2}<5 \lambda / 2$ and the solid line shows the distance of $X_{2}$ from $f\left(x_{1}\right)$ when $x_{2}$ minimises $P(x)$ over the range $f\left(x_{1}\right)-\lambda / 3<x_{2}<f\left(x_{1}\right)+\lambda / 3$. The dotted line is the graph of $C\left(\delta^{*}\right)^{1 / 2}$, where $C>0$ is a constant. 